\title{
Aromatherapy in Patients with Cardiovascular Diseases: A Systematic Review
}

\author{
Lissandra de Souza Lopes, ${ }^{1,5}$ Daiana Bündchen, ${ }^{2}$ Felipe Cardozo Modesto, ${ }^{1,3}$ Monica Quintão, ${ }^{1,3}$ Sergio \\ Chermont, ${ }^{1,4,5}$ Ana Carla Dantas Cavalcanti, ${ }^{1,5}{ }^{\oplus}$ Evandro Tinoco Mesquita ${ }^{1}$ \\ Universidade Federal Fluminense, ${ }^{1}$ Niterói, $R J$ - Brazil. \\ Universidade Federal de Santa Catarina, ${ }^{2}$ Florianópolis, SC - Brazil. \\ Instituto Nacional de Câncer, ${ }^{3}$ Rio de Janeiro, RJ - Brazil. \\ Hospital Santa Marta - Niterói, ${ }^{4} R J$ - Brazil. \\ Clinica de Insuficiência Cardíaca Coração Valente - UFF, ${ }^{5}$ Niterói, RJ - Brazil
}

\section{Abstract}

Background: Aromatherapy consists in the use of volatile aromatic compounds of plant essential oils. Application methods include massage, baths, and mainly inhalation. Lavender essential oil is considered the most effective treatment for emotional disorders, such as stress and anxiety, due to its anxiolytic and sedative agents, which are known to interfere with physiological cardiovascular reactions.

Objectives: To investigate the effects of aromatherapy using lavender essential oil on hemodynamic responses and emotional aspects of patients with cardiovascular diseases.

Methods: A systematic review was conducted using Embase, Bireme, MEDLINE, PEDro, and Scopus electronic databases. Randomized clinical trials that evaluated hemodynamic and emotional outcomes using interventions with lavender essential oil in patients with cardiovascular diseases were selected. Of 539 studies initially identified, 51 were read in full and only 5 were eligible for inclusion.

Results: Reductions were demonstrated in hemodynamic responses, such as systolic and diastolic blood pressure, mean arterial pressure, and heart rate, as well as a decrease in anxiety, depression, stress, and fatigue compared with the control group. Statistical significance was set at $p<0.05$.

Conclusion: Aromatherapy with the use of lavender essential oil provided benefits to hemodynamic parameters, such as anxiety, stress, depression, and fatigue levels, in patients with cardiovascular diseases. (Int J Cardiovasc Sci. 2021; 34(1):74-80)

Keywords: Cardiovascular Diseases; Aromatherapy; Systematic Review; Anxiety; Lavandula; Hemodynamics.

\section{Introduction}

Cardiovascular diseases have the highest incidence of morbidity and mortality in the world. According to the World Health Organization (WHO), they caused approximately 17.9 million deaths in 2016, accounting for $31 \%$ of all causes of death worldwide. ${ }^{1}$ Stress and anxiety seem to destabilize cardiovascular regulation, adversely affecting the autonomic and neuroendocrine pathways and, consequently, cardiovascular parameters and tissue perfusion. ${ }^{2,3}$ Recently, Ma et al., ${ }^{4}$ observed that negative emotions can increase systolic blood pressure (SBP), diastolic blood pressure (DBP), heart rate (HR), and cortisol levels, whereas positive emotions can decrease SBP and HR.

In this context, complementary therapies to control stress and anxiety have been studied, and integrative practices have shown to be promising resources in promoting health to patients with chronic diseases, including cardiovascular disease. ${ }^{5}$ In Brazil, a decision by the National Policy and Integrative and Complementary Practice (PNPIC) was approved within the scope of 
the Brazilian public unified health system (SUS), and Ordinance No. 702 published on March 21, 2018, added 12 new practices, including aromatherapy, which consists in the use of essential oils extracted from aromatic plants ${ }^{6}$ and is commonly administered by inhalation or massage. ${ }^{7}$

Lavender, with the scientific name of Lavandula, has been presented as a relevant substrate in the practice of aromatherapy. Its oil is extracted from its flower, and the main chemical compounds are linalool and linalyl acetate. ${ }^{8,9}$ These compounds are rapidly absorbed by the skin and detected in the bloodstream after topical application, reaching peak levels in approximately 19 to 20 minutes after application. ${ }^{8,10}$ Inhalation starts in the nose, where there is a chain of chemoreceptor olfactory neurons. Stimuli are conducted from the olfactory bulb, taking information to the cerebral cortex, hypothalamus, and hippocampus, connecting to the limbic system. ${ }^{5,11}$ Because of its anxiolytic and sedative properties, the Lavandula Officinalis species is recognized for its effective treatment of stress and anxiety..$^{10-12}$

The purpose of this review was to investigate the effects of the use of lavender essential oil on hemodynamic responses and emotional aspects in patients with cardiovascular diseases.

\section{Methods}

This systematic review followed the Preferred Reporting Items for Systematic Reviews and MetaAnalysis (PRISMA) guidelines. ${ }^{13}$ The research question was developed using the PICO strategy, where participants $(P)$ were patients with cardiovascular diseases, the intervention (I) was aromatherapy with the use of lavender essential oil, the comparison (C) was randomized controlled studies, and the outcome (O) was hemodynamic and emotional responses to the intervention. Question: What are the effects of aromatherapy with lavender essential oil on hemodynamic response and emotional aspects in patients with cardiovascular diseases?

\section{Eligibility Criteria for Study Design}

The inclusion criteria applied in the present review are shown in Table 1 . The primary outcome was the analysis of hemodynamic responses, such as SBP, DBP, HR, and respiratory rate (RR). Secondary outcomes included emotional responses, such as anxiety, stress, fatigue, and depression.

\section{Table 1 - Inclusion Criteria}

Study Design

- Randomized Clinical Trials

Participants

- Both sexes with cardiovascular disease

Intervention

- Use of lavender essential oil

- Application of aromatherapy with inhalation and massage

- Measurement of hemodynamic parameters

- Measurement of emotional responses

Results Measured

- Intervention of aromatherapy versus hemodynamic responses

- Intervention of aromatherapy versus emotional factors

Comparison

- Intervention of aromatherapy with inhalation versus control

- Intervention of aromatherapy with massage versus control

\section{Data Sources}

The search was performed in May 2020, with no publication date restrictions, using the following 5 databases: Medical Literature Analysis and Retrieval System Online (MEDLINE), Virtual Health Library (Bireme), Physiotherapy Evidence Database (PEDro), Embase, and Scopus.

\section{Search Strategy}

The search strategy used MeSH or DeCs terms, combined by the Boolean operators "AND" and "OR", as well as other descriptors with similar concepts: aromatherapy and Lavandula, and heart or hemodynamics or cardiovascular diseases.

\section{Study Selection and Data Extraction}

Two reviewers screened titles and abstracts independently. Potentially relevant studies were retrieved for full-text reading. After reading the full text, all studies that met the inclusion criteria were selected for review. Any disagreement in the studies would be resolved by consulting a third reviewer for arbitration, which was not necessary.

\section{Quality Assessment}

The Cochrane Collaboration's tool was used for assessing methodological quality and risk of bias. This tool has 7 domains: 1 . Generation of the Random 
Allocation Sequence; 2. Allocation Concealment; 3. Blinding of Participants and Health Professionals; 4. Blinding of Outcome Assessors; 5. Incomplete Outcome Data; 6. Selective Outcome Reporting; and 7. Other Sources of Bias. ${ }^{14}$ Each domain was characterized according to the risk of bias of the studies. The assessment was performed independently by 2 reviewers (LSL and FCM).

\section{Results}

Of 539 articles initially identified through the search strategy, 213 were from MEDLINE, 113 from Bireme, 10 from Embase, and 203 from Scopus. No studies were identified in the PEDro database. A total of 80 articles were excluded because they were duplicates and 408 after reviewing titles and abstracts, resulting in a set of 51 articles for full-text reading. After independent analysis by the reviewers, 46 were excluded for not meeting the inclusion criteria (Table 1), resulting in 5 studies included in our systematic review. A flowchart of the study selection process is shown in Figure 1.

All 5 studies used aromatherapy with lavender essential oil in patients with cardiovascular diseases. ${ }^{12,15-18}$ The studies were published from 1992 to 2020. The sample size ranged from 60 to 135 patients, ${ }^{15,17}$ and the mean age was 60 to 73 years. ${ }^{16,17}$ Three studies ${ }^{12,15,16}$ included participants of both sexes, and 2 studies ${ }^{17,18}$ included only women. Regarding the participants, 2 studies $^{12,15}$ selected patients who underwent coronary artery bypass graft surgery, 1 study ${ }^{16}$ assessed patients with coronary artery bypass graft preoperatively, and 2 studies ${ }^{17,18}$ evaluated a sample of women with acute coronary syndrome.

Three studies ${ }^{12,15,16}$ administered the intervention by inhalation and 2 studies ${ }^{17,18}$ applied massage associated

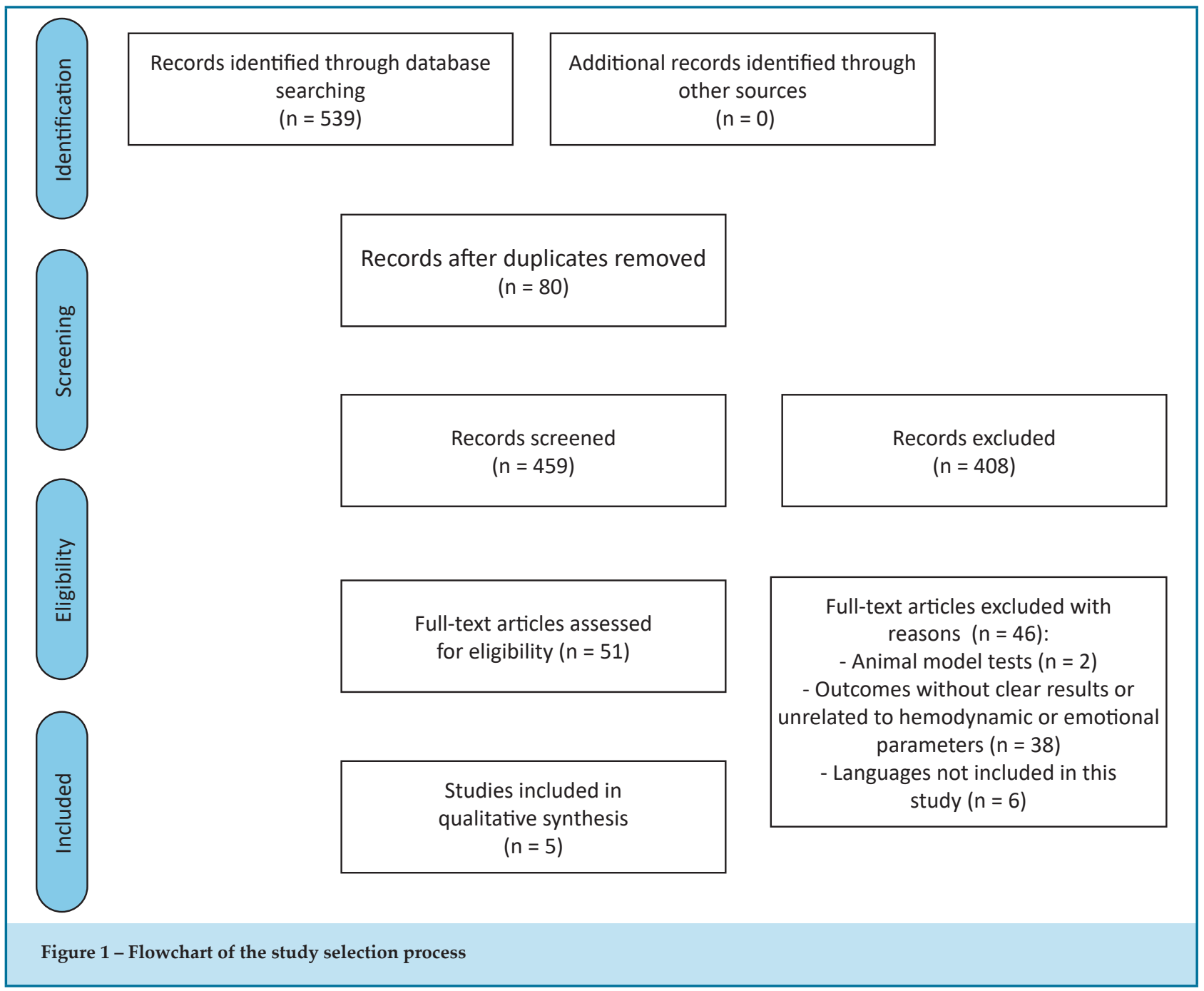


with reflexology (technique that stimulates different points on the feet). In 4 studies, ${ }^{12,15-17}$ intervention lasted for 20 minutes; 1 study ${ }^{18}$ did not report total intervention duration. All studies included in this review used a control group. Two studies ${ }^{12,16}$ completed the intervention on days 2 and 3 after surgery. The other 3 studies $^{16-18}$ completed the intervention in 1 day.

Five studies ${ }^{12,15-18}$ evaluated hemodynamic responses such as SBP, DBP, HR, and RR. Two studies ${ }^{17,18}$ included mean arterial pressure (MAP). Besides hemodynamic parameters, 1 study ${ }^{18}$ evaluated $\mathrm{SaO}_{2}$ and 2 studies ${ }^{12,15}$ evaluated body temperature. Regarding emotional aspects, 2 studies evaluated only the level of anxiety, ${ }^{12,16}$ 1 study $^{18}$ evaluated anxiety and depression levels, 1 study evaluated fatigue levels, ${ }^{17}$ and 1 study investigated stress. ${ }^{15} \mathrm{~A}$ p-value $<0.05$ was considered significant in all studies. The characteristics of the included studies are shown in Table 2.

In the study by Seifi et al., ${ }^{12}$ there were no statistically significant reductions in HR and RR or in anxiety levels; however, a difference in SBP and DBP was observed on the third day of intervention (30 minutes after intervention) between the aromatherapy and control groups.

Bikmoradi et al., ${ }^{15}$ reported different levels of stress between the aromatherapy and control groups 3 days after surgery. Regarding hemodynamic variables, there were no differences in relevant statistics, and the results were presented with the confidence interval.

Rajai et al., ${ }^{16}$ observed reduced levels of anxiety after intervention in the aromatherapy group; however, no significant reduction was observed in stress. Regarding hemodynamic responses, a reduction in HR was observed after intervention in the aromatherapy group compared with the control group.

Bahrami et al., ${ }^{17}$ reported a decrease in fatigue levels from severe to moderate and considered it a good intervention for the management of fatigue. They also showed a reduction in SBP, MAP, and HR compared with the control group.

In a second study, Bahrami et al., ${ }^{18}$ observed a significant reduction in anxiety and depression levels in the aromatherapy group compared with the control group. Regarding hemodynamic parameters, there were significant reductions in SBP, DBP, MAP, and HR.

Regarding study quality, all studies included in this review showed an appropriate design and carefully assessed the processes of randomization and group allocation, most of them presenting a blind assessment of the results to reduce the risk of bias. In the included studies, there were difficulties in keeping participants blind to the application of scent and massage, but there was no risk of bias due to the allocation of intervention in either group. The studies reported the outcomes as complete outcome data or reported the losses to follow-up.

\section{Discussion}

This review demonstrated that the topic of cardiovascular response to aromatherapy has not been fully explored, as there are only a few studies with a design that supports the use of aromatherapy with lavender essential oil as a therapeutic resource in the control of hemodynamic responses and emotional aspects in patients with cardiovascular diseases. Nevertheless, the evidence presented in this review shows the relevant effects of lavender essential oil use on hemodynamic responses, such as SBP, DBP, MAP, and HR, as well as on the reduction of stress, anxiety, and fatigue, when compared with control groups. ${ }^{19,20}$

In a study using a rabbit model, Koto et al., ${ }^{21}$ found that linalyl acetate produces progressive effects during vascular contraction by observing the relaxation of the carotid artery and showed that linalyl acetate is the main component of lavender. However, some opposing studies pointed out linalool as the most relevant active substance. ${ }^{8,9,11}$ This can be attributed to the different botanical species of Lavandula and its variations, since they are directly influenced by the location and the climate where they are cultivated, and this influences the chemical structure of the essential oil. ${ }^{8}$ Studies included in this review did not mention the lanvandula species used in the interventions.

The use of lavender essential oil triggered some cardiovascular responses, and these responses are attributed to the emotional effects of lavender and may be associated with reduced levels of stress and anxiety, which regulate mood and emotion by stimulating the limbic system. ${ }^{5,10}$ This hypothesis is supported by the findings of Koulivand et al., ${ }^{8}$ who reported that lavender causes therapeutic effects by activating the limbic system, stimulating emotions, and activating the amygdala and the hippocampus, responsible for behavior and memory. This is in line with the results of the present review which showed a reduction in anxiety, stress, and fatigue.

Hosseni et al., ${ }^{19}$ reported that aromatherapy has effects on SBP, in addition to the effects on HR reduction. A reasonable explanation could be that described by 


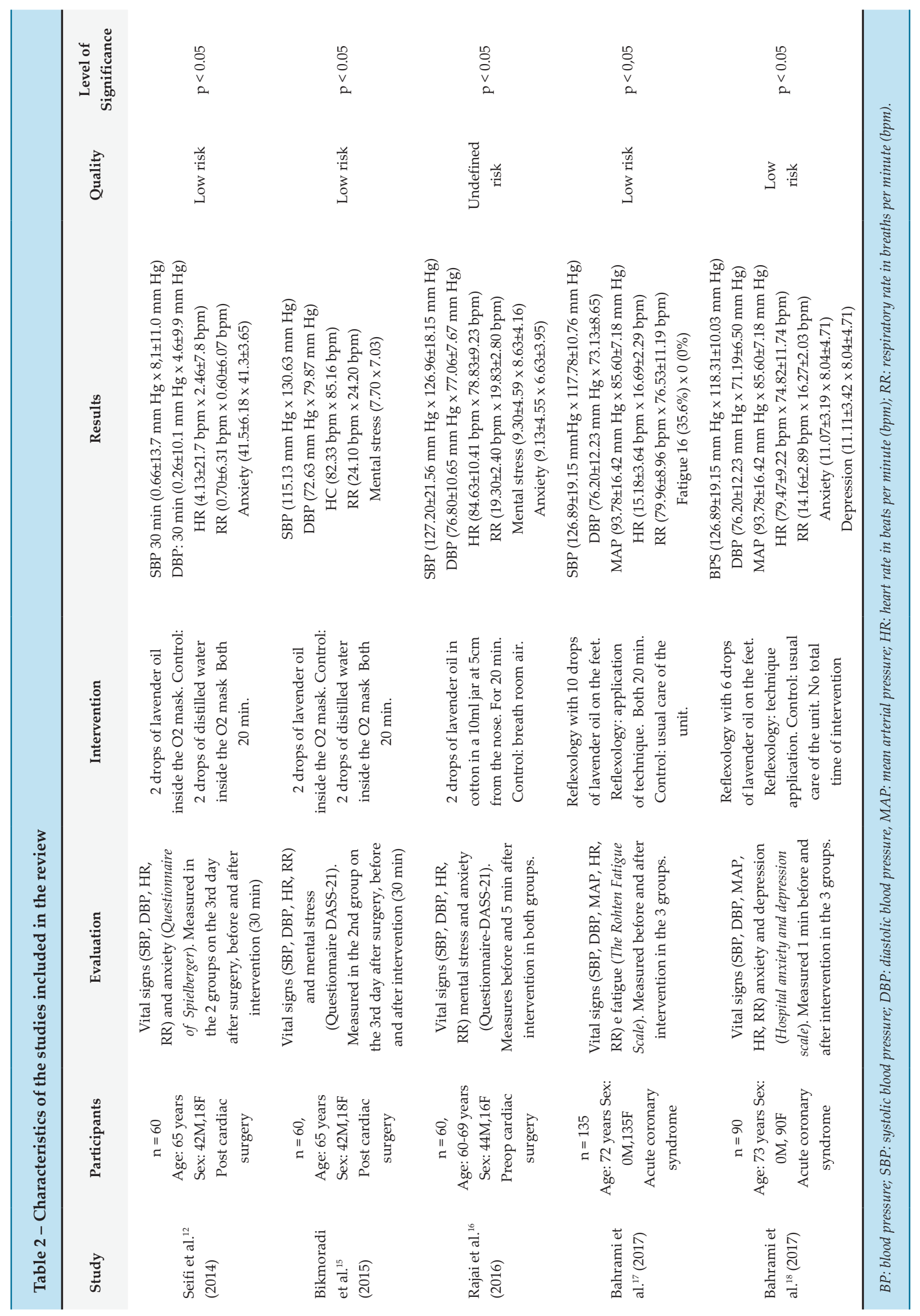


Henz et al., ${ }^{10}$ in which they state that the action of linalool in reducing SBP could be related to the effects of sympathetic deactivation of the autonomic nervous system. This hypothesis can be supported by the studies of Rajai et al., ${ }^{16}$ and Bahrami et al., ${ }^{18}$ who found a reduction in HR in the aromatherapy intervention groups. Also, the reduction in anxiety levels observed by Rajai et al., ${ }^{16}$ and Bahrami et al., ${ }^{18}$ may be attributed to the anxiolytic and sedative effects of linalool on the autonomic nervous system as a parasympathetic mimetic effect. ${ }^{20}$ Koulivand et al., ${ }^{8}$ demonstrated similar anxiolytic action in an animal experiment comparing linalool with chlordiazepoxide.

Shiina et al., ${ }^{20}$ and Bikmoradi et al., ${ }^{15}$ observed significant reductions in cortisol levels, whereas Bahrami et al., ${ }^{18}$ showed significant reductions in the levels of anxiety and depression, which were justified by the relaxing effects of lavender on the autonomic nervous system. Hosseni et al., ${ }^{19}$ reported that anxiety increases cortisol and adrenaline levels, generating a stress response in the body. Bahrami et al., ${ }^{17}$ in their first study, assessed fatigue levels and found a reduction from severe to moderate after aromatherapy.

In both studies conducted by Bahrami et al., ${ }^{17,18}$ foot massage was used as an intervention resource. In this respect, the authors reported that topical massage is absorbed by the skin and stimulates the olfactory system, affecting the limbic system and stimulating the parasympathetic nervous system. Consequently, there is a stabilization of metabolic and physiological parameters, leading to relaxation. Henz et al., ${ }^{10}$ reported that the absorption of linalool through massage could be psychologically more relaxing than inhalation.

A limitation of this study is that, although most of the included studies have an adequate design and low risk of bias, there is a small number of studies on this topic. Also, differences in the application protocols and the heterogeneity of the study populations made it impossible to pool data for meta-analysis.

\section{Conclusion}

This systematic review showed that aromatherapy using lavender essential oil has beneficial effects on hemodynamic parameters. Despite the scarce evidence, aromatherapy appears to control the levels of anxiety, stress, depression, and fatigue in patients with cardiovascular diseases. However, there remains a gap in knowledge and more evidence is needed on the use of aromatherapy and its effects on cardiovascular diseases. Further research on the effects of aromatherapy with lavender essential oil in the treatment of patients with cardiovascular diseases is required to explain the physiological mechanisms involved in the results found in this review and to support the clinical practice of aromatherapy as a complementary activity that determines the improvement of health and quality of life in patients with cardiovascular diseases.

\section{Author Contributions}

Conception and design of the research: Lopes LS, Tinoco EM, Chermont S. Acquisition of data: Lopes LS, Modesto F. Analysis and interpretation of the data: Lopes LS, Bündchen DC, Quintão M, Chermont S. Statistical analysis: Lopes LS, Bündchen DC, Chermont S. Writing of the manuscript: Lopes LS, Tinoco EM, Chermont S. Critical revision of the manuscript for intellectual content: Lopes LS, Bündchen DC, Quintão M, Modesto F, Tinoco EM, Chermont S.

\section{Potential Conflict of Interest}

No potential conflict of interest relevant to this article was reported.

\section{Sources of Funding}

There were no external funding sources for this study.

\section{Study Association}

This study is not associated with any thesis or dissertation work. 


\section{References}

1. Organização Mundial de Saúde. (OMS) [homepage na internet]. Ficha Técnica sobre Doenças Cardiovasculares [acesso em 22 de agosto 2017]. Disponível em https://www.who.int/news-room/fact-sheets/detail/ cardiovascular-diseases-(cvds).

2. Ginty AT, Kraynak ET, Fisher JP, Gianaros PJ. Cardiovascular and autonomic reactivity to psychological stress: Neurophysiological substrates and links to cardiovascular disease. Auton Neurosci.2017 Nov; 207:2-9.

3. Cohen BE, Edmondson D, Kronish IM. State of the art review: depression, stress, anxiety, and cardiovascular disease. Am J Hypertens. 2015 Nov;28(11):1295-302.

4. Ma L, Li Y, Feng M. Positive emotion and cardiovascular disease in elderly people. Int J Clin Exp Med. 2015 May;8(5):6682-6

5. Santos MVJ, Rosa CG, Santos PS, Raush PC, Bellinati NVC. Práticas Integrativas na Promoção à Saúde em Doenças Crônicas: Uma Revisão de Literatura. Rev Interdiscipl Estudos Saúde.2019;(2):41-56.

6. Brasil. Ministério da Saúde. Gabinete do Ministro - PORTARIA № 971, DE 21 MARÇO DE 2018. Altera a Portaria de Consolidação nº 2/GM/ MS, de 28 de setembro de 2017, para incluir novas práticas na Política Nacional de Práticas Integrativas e Complementares - PNPIC. [acesso em 14 de maio de 2020] Disponível em :https://bvsms.saude.gov.br/bvs/ saudelegis/gm/2018/prt0702_22_03_2018.html

7. Posadzki P, Alotaibi A, Edzard E. Adverse effects of aromatherapy: A systematic review of case reports and case series. Int J Risk \& Saf Med. 2012 Jan;1;24(3):147-61.

8. Brito AMG, Rodrigues SA, Brito RG, Xavier LF. Aromaterapia: da gênese a atualidade. Rev Bras Plantas Med. 2013;15(4):789-93

9. Koulivand P, Ghadiri M, Gorji A Lavender and the Nervous System. Evid Based Complement Alternat Med. 2013 Mar;2013:681304

10. Salamanti A, Mashouf S, Mojab F. Effect of Inhalation of Lavender Essential Oil on Vital Signs in Open Heart Surgery ICU. Iran J Pharm Res. 2017 Winter;16(1):404-9.

11. Herz RS. Aromatherapy facts and fictions: a scientific analysis of olfactory effects on mood, physiology and behavior. Int J Neurosci. 2009;119(2):263-90.

12. Milanos S, Elsharif S.A, Janzen D, Buettner A, Villmann C. Metabolic Products of Linalool and Modulation of GABAA Receptors. Front Chem. 2017;5(46)1-9

13. Seifi Z, Bikmoradi A, Oshvandi K, Poorolajal J, Araghchian M, Safiaryan R. The effect of lavender essential oil on anxiety level in pacients undergoing coronary artery bypass surgery: A double- blinded randomized clinical trial. Iran J Nurs Midwifery Res. 2014 Nov;19(6):574-80

14. Galvão TF, Pansani TSA, Harrad D. Principais itens para relatar Revisões sistemáticas e Meta-análises: A recomendação PRISMA*. Epidemiol Serv Saúde. 2015 Abr-Jun;24(2):335-342

15. Carvalho APV, Silva V, Grande AJ. Avaliação do risco de viés de ensaios clínicos randomizados pela ferramenta da colaboração Cochrane. Diagn Trat. 2013;18(1):38-44.

16. Bikmoradi A, Seifi Z, Poorolajal J, Araghchian M, Safiaryan R, Oshvandi K. Effect of inhalation aromatherapy with lavender essential oil on stress and vital signs in patients undergoing coronary artery bypass surgery: a single-blinded randomized clinical trial. Complem Ther Med.2015 Jun;23(3):331-8.

17. Rajai N, Sajadi S, Teymouri F, Zareiyan A, Siavoshi S, Malmir M. The effect of aromatherapy with lavender essential oil on anxiety and stress in pacients undergoing coronary artery bypass graft surgery. Jundishapur J Chronic Dis Care. 2016 Oct;5(4):e34035.

18. Bahrami T, Rejeh N, Karimooi MH, Vaismoradi M, Tadrisi SD and Sieloff CL. Aromatherapy massage versus reflexology on female elderly with acute coronary syndrome. Murs Crit Care. 2018 Sep;23(5):229-36.

19. Bahrami T, Rejeh N, Karimooi M.H, Vaismoradi M, Tadrisi S.D, Sieloff C. Effect of aromaterapy massage on anxiety, depression, and physiologic parameters in older patients with acute coronary syndrome: A randomized clinical trial. Int J Nurs Pract. 2017 Dec;23(6)1-10.

20. Hosseini S, Heydari A, Vakili M, Moghadam S, Tazyky S. Effect of lavender essence inhalation on the level of anxiety and blood cortisol in candidates for open-heart surgery. Iran J Nurs Midwifery Res. 2016 Jul-Aug;21(4):397-401.

21. Shiina Y, Funabashi N, Lee K, Toyoda T, Sekine T, Honjo S, Hasegawa R, Kawata T, Wakatsuki Y, Hayashi S,Murakami S, Koike K, Daimon M, Komuro I. Relaxation effects of lavender aromatherapy improve coronary flow velocity reserve in healthy men evaluated by transthoracic Doppler echocardiography. Int J Cardiol.2008 Sep 26;129(2):193-7.

22. Koto R, Imamura M, Watanabe C, Obayashi S, Shiraishi M, Sasaki Y, Azuma H. Linalyl Acetate as a Major Ingredient of Lavender Essential Oil Relaxes the RabbitVascular Smooth Muscle through Dephosphorylation of Myosin Light Chain. J Cardiovasc Pharmacol. 2006 Jul;48(1):850-6.

23. Najafi Z, Taghadosi M, Sharifi K, Farrokhian A, Tagharrobi Z. The Effects of Inhalation Aromatherapy on Anxiety in Pacients With Myocardial Infarction: A Randomized Clinical Trial. Iran Red Crescent Med J. 2014 Aug;16(8):e15485. 